\title{
Mitochondrial DNA polymorphisms in Andersen-Tawil syndrome
}

\author{
Armando Totomoch-Serra'1,2, Cesar A. Brito-Carreón', Maria de L. Muñoz', \\ David Cervantes-Barragan ${ }^{3}$, Manlio F. Márquez ${ }^{4}$ \\ 1 Department of Genetics and Molecular Biology, Center for Research and Advanced Studies of the National Polytechnic Institute (CINVESTAV-IPN), Mexico City, Mexico \\ 2 PhD Program in Medical Sciences, University of the Frontier (UFRO), Temuco, Chile \\ 3 Department of Genetics, South Central High Specialty Hospital of Mexican Petroleum (PEMEX), Mexico City, Mexico \\ 4 Department of Cardiac Electrophysiology, National Institute of Cardiology "Ignacio Chávez," Mexico City, Mexico
}

Correspondence to: Manlio F. Márquez, MD, Department of Cardiac Electrophysiology, National Institute of Cardiology "Ignacio Chávez," Juan Badiano 1, Sección XVI. Tlalpan 14010, Ciudad de México, Mexico, phone: +525555133740 , email: manlio.marquez@cardiologia.org.mx Received: December 10, 2019. Revision accepted: March 31, 2020.

Published online: April 2, 2020. Kardiol Pol. 2020; 78 (6): 581-583 doi:10.33963/KP.15274 Copyright by the Author(s), 2020
Introduction Andersen-Tawil syndrome (ATS) is a heart rhythm disorder classified as type 7 long QT syndrome and characterized by muscular, neurological, and skeletal involvement. So far, mutations in the KCNJ2 (60\% of cases) and $K C N J 5$ ( $<1 \%$ of cases) genes have been reported to be the underlying cause of the disease.

In addition to the classic triad of periodic paralysis, ventricular arrhythmias, and mild-to-moderate dysmorphism, some case reports also noted deficits in executive functioning skills, abstract reasoning, ${ }^{1}$ and seizures, ${ }^{2}$ which indicates that ATS affects the central nervous system. The clinical variability observed in the heart, skeletal muscles, and the brain could be a result of incomplete penetrance or interaction of modifier genes, as seen in epilepsy. ${ }^{3}$ Given the background, mitochondrial DNA (mtDNA) polymorphisms can influence the phenotype by altering the energy demand of the tissues affected in the triad of ATS.

The human mitochondrial genome includes 16569 base pairs (bps). They form a maternally inherited, double-stranded, circular DNA chain, which encodes 13 polypeptides-components of the respiratory chain complexes I, III, IV, and $\mathrm{V}$. This genome is characterized by a high rate of mutations, which regularly affect coding sequences. The hypervariable sites I and II (HVR-I and HVR-II, respectively) are part of the D-loop, a triple-helix structure consisting of $1100 \mathrm{bps}$, which is located in mtDNA. The HVR-I site (approximately 400 bps) is the most polymorphic, informative, and mutational hotspot used previously. ${ }^{4}$

Regarding heart rhythm disorders, Stocchi et $a l,{ }^{5}$ who sequenced whole mitochondrial genomes, suggested that a large number of polymorphisms in mtDNA may be associated with more severe Brugada syndrome.

Considering that the heart, skeletal muscles, and the brain use a high number of mitochondria and that these tissues are essential components affected in the ATS triad, mtDNA polymorphisms can be expected to partially influence the disease phenotype.

In this article, we present results of our study aimed to identify higher mtDNA polymorphisms in HVR-I in 5 patients with ATS.

Methods Recruitment of participants Five study subjects together with their 4 mothers and 3 fathers were enrolled. Based on clinical evaluation and electrocardiographic examination, all study participants met the diagnostic criteria for ATS and were inhabitants of the states of the central and north central regions of Mexico. Patients 1, 2, and 3 were included owing to the presence of arrhythmia, history of limb weakness, facial features, and no history of sudden cardiac death. Patient 4 was enrolled because of sudden death of her twin sister and referred to the National Institute of Cardiology (Instituto Nacional de Cardiologia) because of suspicion of fatal arrhythmia. ${ }^{2}$ The ATS phenotype was identified based on the presence of arrhythmia and long QT syndrome, the analysis of the facial phenotype, and history of sudden death in the family. Patient 5 was included in the study but showed no cardiac involvement. The institutional review board approved the study, and the participants provided written informed consent. 
Genomic DNA extraction Five milliliters of whole blood were obtained from each patient, and DNA was extracted using the salting-out method.

The DNA integrity was assessed in a $1 \%$ agarose gel and quantified with the NanoDrop 2000 Spectrophotometer (Thermo Fisher Scientific, Waltham, Massachusetts, United States) by measuring the ratio of absorbance at $260 \mathrm{~nm}$ and 280 $\mathrm{nm}$ (approximately 1.8 accepted as appropriate).

Genotyping nuclear DNA Genetic testing for ATS verification was sponsored in 1 patient and performed with the use of massive parallel sequencing for the KCNJ2, CACNA1C, and KCNQ1 genes, provided by Sistemas Genómicos (Valencia, Spain). In the remaining 4 patients with ATS, DNA was analyzed by Sanger sequencing for the KCNJ2 gene, including all exons and intron/ exon borders. When ATS was identified, cascade genetic screening was performed in the relatives who could participate in the study.

Genotyping mitochondrial DNA In order to identify polymorphic positions in patients with ATS, the HVR-I region of mtDNA (400 bps) was amplified and sequenced by the Sanger method using the 5'-ATACACCAGTCTTGTAAACC-3' and 5'-CACGGAGGATGGTGGTCAAG-3' primers. All the sequences obtained were aligned, and polymorphisms were identified using the DnaSP 5.10.1 (Universitat de Barcelona, Barcelona, Spain) and MEGA 4.0 (Center of Evolutionary Functional Genomics Biodesign Institute, Arizona State University, Arizona, United States) software.

Statistical analysis Genotype frequencies were estimated by direct counting and distribution. Categorical variables were analyzed using the Fisher exact or $\mathrm{X}^{2}$ tests and performed with the R software, version 3.6.3 (R Foundation for Statistical Computing, Vienna, Austria).

A $P$ value less than 0.05 was considered significant. A 2-dimensional scaling plot was drawn with the RStudio Desktop 1.2.5033 (RStudio, Inc., Boston, Massachusetts, United States) software using data from $250 \mathrm{bps}$ of the HVR-I region and the Kimura 2-parameter distance values obtained from the sequences (based on transition and transversion substitution rates), which summarized genetic distances between the study patients and their fathers. The Cambridge Reference Sequence was used as a reference sequence in this study (GenBank number, NC_012920.1).

Results and discussion Genetic testing Five study participants were identified as carrying the following mutations located in the KCNJ2 gene: p.Ser136Phe (Patient 1), p.Gly144Ala (Patient 2), p.Arg218Trp (Patients 3 and 4), and p.Asp71Val (Patient 5) (table 1).
Polymorphisms in the HVR-I site The polymorphisms identified in 250 bps of the HRV-I site are shown in Supplementary material, Table S1. In the study patients with ATS, 14 polymorphisms were found and compared with the Cambridge reference sequence. A significant difference $(P=0.04)$ was found between patients and their healthy fathers after comparing polymorphism distribution. Concordance was seen between the $\mathrm{T} 16189 \mathrm{C}$ polymorphisms in 3 patients and the C16223T, C16290T, and G16319A polymorphisms in 2 individuals.

Multidimensional scaling plot A multidimensional scaling plot (Supplementary material, Figure S1) was established to identify an ancestral relationship among patients and fathers included in the study. It showed that the mtDNA sequences of the study patients and their fathers were dispersed and no relationship was found among them. However, we observed a slight clustering between Patient 1 (p.Ser136Phe) and $\mathrm{Pa}$ tient 4 ( $\mathrm{p}$ Arg218Trp). The distance between their places of origin was approximately 340 kilometers, and the ancestral relationship was unclear owing to lack of longer mitochondrial DNA sequences. The polymorphisms identified in the mothers were similar to those in the study patients.

The prevalence of ATS is unknown because of the limited number of patients diagnosed worldwide. Fortunately, in the last 10 years, a growing number of cases with their clinical characteristics have been reported in the literature. Considering the high density of mitochondria in the heart, the brain, and skeletal muscles, we studied the HVR-I site (which is highly polymorphic) located in the mitochondrial genome to identify mtDNA polymorphisms in ATS and compare them with mtDNA polymorphisms in patients' fathers who could take part in the study. The results obtained were in line with previous studies, in which complete mitochondrial genomes were sequenced in patients with Brugada syndrome and more polymorphisms were found. ${ }^{5}$ The difference in the distribution of mtDNA polymorphisms may be indicative of a haplotype having more significant effects on the synthesis of mitochondrial proteins.

Of note, the diagnosis of ATS without cardiac abnormalities remains a challenge. The family history of sudden death in some patients with ATS highlights the need for further studies on the KCNJ2 gene mutations in the context of modifying factors and the necessity to use cardioverter-defibrillators in this population. ${ }^{6,7}$ Altered protein synthesis in mitochondria, being a consequence of polymorphisms in specific sites, may affect mitochondrial biogenesis leading to the decreased effectiveness of the respiratory chain and triggering acute episodes of limb weakness. ${ }^{8}$ Further studies on mitochondrial 
TABLE 1 Clinical characteristics of patients with Andersen-Tawil syndrome and their relatives

\begin{tabular}{llllll} 
Family status & Sex & Mutation & LQT & Facial phenotype & $\begin{array}{l}\text { Muscular } \\
\text { involvement }\end{array}$ \\
\hline Patient 1 & F & p.Ser136Phe & No & No & Yes \\
\hline Patient 2 & F & p.Gly144Ala & No & Yes & Yes \\
\hline Patient 3 & M & p.Arg218Trp & No & Yes & Yes \\
\hline Patient 4a & F & p.Arg218Trp & Yes & Yes & Yes \\
\hline Patient 5 & F & p.Asp71Val & No & Yes & Yes \\
\hline Mother 1 & F & - & No & No & No \\
\hline Mother 2 & F & - & No & No & No \\
\hline Mother 3 & F & - & No & No & No \\
\hline Mother 4 & F & p.Arg218Trp & Yes & Yes & Yes \\
\hline Father 2 & M & - & No & No & No \\
\hline Father 3 & M & - & No & No & No \\
\hline Father 4 & M & - & No & No & No \\
\hline
\end{tabular}

a The patient participated in a previous study by Márquez et al. ${ }^{2}$

Abbreviations: $F$, female; $M$, male; LQT, long QT interval

dynamics, regulatory pathways, and interaction between mitochondrial DNA with nuclear DNA in ATS are needed.

Research on ATS has captured the attention of basic and clinical scientists in recent years. However, it is still considered an exceptionally rare disease because of its infrequent identification at the tertiary care level. The analysis of extracardiac manifestations and their modifying genetic factors are issues currently investigated in ATS. This study suggested that the implication of mitochondrial polymorphisms and probably haplotypes may be one of the genetic factors influencing the classic triad of ATS.

\section{SUPPLEMENTARY MATERIAL}

Supplementary material is available at www.mp.pl/kardiologiapolska.

\section{ARTICLE INFORMATION}

CONFLICT OF INTEREST None declared.

OPEN ACCESS This is an Open Access article distributed under the terms of the Creative Commons Attribution-Non Commercial-No Derivatives $4.0 \mathrm{In}$ ternational License (CC BY-NC-ND 4.0), allowing third parties to download articles and share them with others, provided the original work is properly cited, not changed in any way, distributed under the same license, and used for noncommercial purposes only. For commercial use, please contact the journal office at kardiologiapolska@ptkardio.pl.

HOW TO CITE Totomoch-Serra A, Brito-Carreón CA, Muñoz ML, et al. Mitochondrial DNA polymorphisms in Andersen-Tawil syndrome. Kardiol Pol. 2020; 78: 581-583. doi:10.33963/KP.15274

\section{REFERENCES}

1 Davies NP, Imbrici P, Fialho D, et al. Andersen-Tawil syndrome: new potassium channel mutations and possible phenotypic variation. Neurology. 2005; 65: 1083-1089. 2 Márquez MF, Totomoch-Serra A, Burgoa JA, et al. Abnormal electroencephalogram, epileptic seizures, structural congenital heart disease, and aborted sudden cardiac death in Andersen-Tawil syndrome. Int J Cardiol. 2015; 180: 206-209. 3 Meisler MH, O'Brien JE, Sharkey LM. Sodium channel gene family: epilepsy mutations, gene interactions, and modifier effects. J Physiol. 2010; 588: 1841-1848.
4 Stoneking M. Hypervariable sites in the mtDNA control region are mutational hotspots. Am J Hum Genet. 2000; 67: 1029-1032.

5 Stocchi L, Polidori E, Potenza L, et al. Mutational analysis of mitochondrial DNA in Brugada syndrome. Cardiovasc Pathol. 2016; 25: 47-54.

6 Mönnig G, Ziaukas V, Koopmann M. Implantable cardioverter-defibrillator therapy in patients with congenital long QT syndrome: do we know what we need to know? Kardiol Pol. 2018; 76: 1665-1667.

7 Stępień-Wojno M, Ponińska J, Rydzanicz M, et al. Sudden cardiac arrest in patients without overt heart disease: a limited value of next-generation sequencing. Pol Arch Intern Med. 2018; 128: 721-730.

8 Auré $\mathrm{K}$, Dubourg 0 , Jardel $\mathrm{C}$, et al. Episodic weakness due to mitochondrial DNA MT-ATP6/8 mutations. Neurology. 2013; 81: 1810-1818. 